\title{
Case Report: Acupuncture And Herbal Treatment With Yunnan Baiyao For Hyperhidrosis
}

\author{
Jihe Zhu ${ }^{1}$,Blagica Arsovska ${ }^{2}$,Kristina Kozovska ${ }^{3}$ \\ ${ }^{1,3}$ Faculty Of Medical Sciences, University Goce Delcev - Shtip, Republic Of Macedonia; \\ ${ }^{2}$ Institute Of Biology, Faculty Of Natural Sciences And Mathematics - Skopje, Republic Of Macedonia;
}

\begin{abstract}
Hyperhidrosis or excessive sweating is a secretion of sweat in bigger amount that the body is needed. The reason of appearance is not clear yet to the Western medicine. Hyperhidrosis can be divided to focal and general and primary and secondary. In the terms of Traditional Chinese Medicine (TCM) hyperhidrosis is called 'spontaneous perspiration' and is seen as disharmony of the protective Qi, a deficiency of Yin or Yang energy. To treat the condition in our case we used acupuncture and herbal treatment. The treated patient is a 16 year old male teenager with excessive sweating on the head, back and stomach for three years and increased in the past one year. The patient has done 16 acupuncture treatments and drank Yunnan Baiyao tea, $0,25 \mathrm{~g}$ once a day in a period of 6 months. The treated points are located on the meridians of Liver, Bladder, Spleen and Kidney. Treatments were done in a clinic for TCM and acupuncture, once a week, with duration of 30-40 minutes in a closed room. Gradually, with the treatments the condition was improved and the body's control of sweating was recovered.
\end{abstract}

Keywords: acupuncture, herbs, sweating, treatment, traditional Chinese medicine

\section{Introduction}

Hyperhidrosis or excessive sweating is a condition of excessive or increased secretion of the sweat glands on different parts of the body. Hyperhidrosis is a very unpleasant condition and visible problem which affects around $3 \%$ of the world population. The condition can cause major psychological difficulties like depression, loss of self-esteem, emotional problems, social isolation, anxiety, discomfort and etc. The body has between two to five million sweat glands and the basic function of the sweating is thermoregulation of the body. Hyperhidrosis can be divided to focal and general and primary and secondary. Focal is when the sweating is localized (for example on soles and hands) and general when the entire body is affected. Primary or idiopathic is when the reason is unknown and the sweating is localized and secondary because of some health condition (tumor, menopause, obesity, diabetes and etc.). [1] [2] The condition affects both women and men equally and usually starts on age before 25. [3] When in puberty, a lot of changes start to happen in the body. It's a period when major changes are happening mostly on the skin, including the sweating, The Western Medicine suggests blockage of the release of acetycholine (neutrotransmitter) with botox - a toxin injection into the sweat glands. [4] The Traditional Chinese Medicine (TCM) has a totally different approach on this matter than the Western Medicine, which totally excludes toxins and chemical substances to be inserted on/into the body. The first choice for treatment of hyperhidrosis is with acupuncture and herbs. The TCM sees the condition as a disharmony of the energy in the body that may be results from deficiency of Yang energy, internal weakness, excessive heat in the body or deficient energy that fails to contain body fluids. The acupuncture and herbal treatment can balance the autonomic nervous system, harmonize the energy and help the body to regain its normal internal state. [5] [6]

\section{Material And Methods}

In this case report is presented a case of teen hyperhidrosis. The treated patient is a 16 year old male teenager with excessive sweating on the head, back and stomach. The condition started three years ago and the sweating increased in the past one year. The patient is an active individual, exercises excessively in gym with normal diagnosis, normal blood pressure, blood sugar levels, appetite, stool, urine, heart and short night time sleeping. The results from the hormonal tests came normal for all hormones including the thyroid. The hyperhidrosis is primary and general, with excessive sweating through the day. The patient has done 16 acupuncture treatments and while the treatments were done he was drinking Yunnan Baiyao (Ginseng) tea in a capsule form $0,25 \mathrm{~g}$ in the morning, per day in a period of 6 months. Treatments were done in a clinic for Traditional Chinese Medicine and acupuncture in Skopje, Macedonia by a doctor specialist in acupuncture. Treatments were done once a week, with duration of 30-40 minutes in a closed room on a room temperature. In the treatment were used fine, sterile acupuncture needles size $0.25 \times 25 \mathrm{~mm}$ produced by Wuijuiang City Medical \& Health Material Co., LTD. Gradually, with the treatments the condition was improved and the 
body's control of sweating was recovered. The treated acupuncture points are located on the meridians of Liver, Bladder, Spleen and Kidney.

\section{Results And Discussion}

Sweating is normal during the puberty period, because the body is susceptible to changes in many ways, but the excessive sweating can be a real problem, especially in a teenager, which in return can cause emotional and psychological problems. Our 16 year old patient responded very well to our therapy. 16 acupuncture treatments were made every week, in a period of 6 months, from $20^{\text {th }}$ June to $3^{\text {rd }}$ November 2016. During the treatments he was drinking Yunnan Baiyao (Ginseng) tea $0,25 \mathrm{~g}$ per day, in the morning. In this case the hyperhidrosis is general, because the excessive sweating was present all over the body, especially on the places around the chest, stomach, back and head. Our theory is that the reasons for the excessive sweating are of some external and internal characters. External - too much exercising and very short time of sleeping, which is why were treated the acupoints located on the meridians of Liver, Bladder and Spleen. Internal - Fear as a factor that affected the Qi and made it damaged. The aim of the treatment was to correct the Qi energy, to balance the energy, to improve the sleeping, stop the excessive sweating and calm the Spirit. According to the TCM theory excessive sweating around the area of head, chest and upper body is a result of Yin or Yang deficiency in the Heart, Kidney and Spleen and accumulation of Dump-Heat. [6] The sweat pores located all over our body are entry and exit for all external pathogens. The problem with the excessive sweating is that not only the external pathogens are thrown out, but also the body essence, concretely the Heart energy is reduced which is considered to be the source of sweating (perspiration). [8] Acupuncture treatment helps to balance and strengthen the sympathetic and parasympathetic nervous systems, as well the herbal treatment, which helps to reduce the inflammation, calm the Heat in the body and diminish the sweating. [7] Yunnan Baiyao is a herb from the Ginseng family and in the treatment is used to clear the heat, resolve the stagnation, invigorate the blood, remove the stasis and tonify the Qi energy. [9] The herb is usually used to stop the bleedings in the gastrointestinal system, but it is also known that alleviates pain, treats blood stasis, promotes normal blood circulation and reduces swelling and inflammation. [10]

\section{Conclusion}

Acupuncture and herbal treatment with Yunnan Baiyao as parts of the Traditional Chinese Medicine are very effective treatments and give positive and satisfying results in the treatment of hyperhidrosis (excessive sweating), improving the condition completely and allowing the body to heal naturally and regain its function.

[1]. Миниќ C.; Прекумерно потење; 2015 [www.betty.mk]

[2]. Nordqvist C.; Hyperhidrosis: Causes, Symptoms, and Effective Treatments; 2016 [www.medicalnewstoday.com]

[3]. Tidy C.; Excessive Sweating (Hyperhidrosis); 2014 [www.patient.info]

[4]. Bouchez C.; Top Teen Skin Problems - and How To Solve Them; 2017 [www.webmd.com]

[5]. Kaufman J.; Hyperhidrosis/Excessive Sweating; 2016 [www.okanaganacupuncture.com]

[6]. Joswick D.; Stop Sweating with Acupuncture; 2017 [www.acufinder.com]

[7]. Zheng L.; Acupuncture \& Hormone Balance; 2013 [www.acuhormone.blogspot.mk]

[8]. Dharmananda S.; On the importance of perspiration in Chinese Medical diagnosis and therapy; The Institute for Traditional Medicine and preventive health care; Basics 2; 1997

[9]. Gruenstern J.; Yunnan Baiyao - Miracle herb for young clinic; IVC Journal; Vol. 4 Issue 1; 2014

[10]. Dharmananda S.; YUNNAN PAIYAO Uses for injury and surgery; gastro-intestinal, respiratory, and urogenital disorders; 2010 [www.itmonline.org] 